\title{
FORMAL INVERSE INTEGRATING FACTORS AND THE NILPOTENT CENTER PROBLEM
}

\author{
ISAAC A. GARCÍA ${ }^{1}$
}

\begin{abstract}
We are interested in deepening knowledge of methods based on formal power series applied to the nilpotent center problem of planar local analytic monodromic vector fields $\mathcal{X}$. As formal integrability is not enough to characterize such a centers we use a more general object, namely, formal inverse integrating factors $V$ of $\mathcal{X}$. Although by the existence of $V$ is not possible to describe all nilpotent centers strata, we simplify, improve and also extend previous results on the relationship between these concepts. We use in the performed analysis the so-called Andreev number $n \in \mathbb{N}$ with $n \geq 2$ associated to $\mathcal{X}$ which is invariant under orbital conjugacy of $\mathcal{X}$. Besides the leading terms in the $(1, n)$-quasihomogeneous expansions that $V$ can have we also prove the following: (i) If $n$ is even and there exists $V$ then $\mathcal{X}$ has a center; (ii) If $n=2$ the existence of $V$ characterizes all the centers; (iii) If there is a $V$ with minimum "vanishing multiplicity" at the singularity then, generically, $\mathcal{X}$ has a center.
\end{abstract}

\section{IntRODUCTION}

An isolated singularity $p_{0} \in \mathbb{R}^{2}$ of a real analytic planar vector field $\mathcal{X}$ is called monodromic if nearby trajectories of $\mathcal{X}$ rotate about $p_{0}$. When the linear part $D \mathcal{X}$ of $\mathcal{X}$ at $p_{0}$ is nonzero the Poincare return map is analytic, hence $p_{0}$ is either a focus or a center. If the eigenvalues of $D \mathcal{X}$ have nonzero imaginary part then, by the classical Poincaré-Lyapunov Theorem, $p_{0}$ is a center if and only if it admits a formal (actually analytic) first integral. In contrast, the formal integrability does not characterize when $p_{0}$ is a nilpotent center, i.e. a center of $\mathcal{X}$ when $D \mathcal{X}$ is nonzero and has two zero eigevalues. In this work we are interested in the nilpotent center problem which consists in characterizing when a nilpotent monodromic singularity $p_{0}$ is a center. There are several techniques designed to attack this problem: (i) blowing-up the singularity by means of generalized polar coordinates (see $[2,9]$ ); (ii) applying normal form theory to orbitally conjugate $\mathcal{X}$, up to some order, into a suitable Liénard normal form as it is explained in [3], and (iii) to embed the nilpotent singularity as the limit of a 1-parameter family of nondegenerate centers (see the initial source [13] until the final corrected version [7]).

The main tool in our approach will be formal inverse integrating factors (see [10] for a survey) instead of formal first integrals.

2010 Mathematics Subject Classification. 37G15, 37G10, 34C25.

Key words and phrases. Monodromic singularity, nilpotent center, integrability, inverse integrating factor.

The author is partially supported by a MINECO grant number MTM2014-53703-P and by a CIRIT grant number 2014 SGR 1204. 
By an affine change of coordinates and a time rescaling an analytic system with a nilpotent singularity can be placed in the form

$$
\dot{x}=y+P(x, y), \quad \dot{y}=Q(x, y),
$$

where $P$ and $Q$ are analytic functions near the origin without constant or linear terms. The following theorem of Andreev characterizes analytic systems (1) for which the origin is monodromic.

Theorem 1 ([4]). For an analytic system of the form (1) with an isolated singularity at the origin let $y=F(x)$ be the unique solution of $y+P(x, y)=0$ such that $F(0)=F^{\prime}(0)=0$ and let

$$
f(x)=Q(x, F(x)) \quad \text { and } \quad \varphi(x)=(\partial P / \partial x+\partial Q / \partial y)(x, F(x)) .
$$

Let $a \neq 0$ and $\alpha \geq 2$ be such that $f(x)=a x^{\alpha}+\cdots$.

When $\varphi$ is not identically zero let $b \neq 0$ and $\hat{\beta} \geq 1$ be such that $\varphi(x)=b x^{\hat{\beta}}+\cdots$. Then the origin of (1) is monodromic if and only if $\alpha=2 n-1$ is an odd integer, $a<0$, and one of the following conditions holds:

(i) $\varphi(x) \equiv 0$

(ii) $\hat{\beta} \geq n$

(iii) $\hat{\beta}=n-1$ and $b^{2}+4 a n<0$.

Definition 2. The Andreev number $n$ of a monodromic singular point at the origin of system (1) is the integer $n \geq 2$ given in Theorem 1 .

In the study of the monodromic nilpotent singularities it is useful and natural to introduce $(1, n)$-quasihomogeneous polynomials.

Definition 3. A polynomial $p_{k} \in \mathbb{R}[x, y]$ is a $(1, n)$-quasihomogeneous polynomials of weighted degree $k$ if $p_{k}\left(\lambda x, \lambda^{n} y\right)=\lambda^{k} p_{k}(x, y)$ for all $\lambda \in \mathbb{R}$. In consequence we get $p_{k}(x, y)=\sum_{i+n j=k} a_{i j} x^{i} y^{j}$ for certain coefficients $a_{i j} \in \mathbb{R}$. On the other hand, a vector field $\mathcal{X}_{i}=p_{i+1} \partial_{x}+q_{i+n} \partial_{y}$ is a $(1, n)$-quasihomogeneous polynomial vector field of weighted degree $i$ if its components $p_{i+1}$ and $q_{i+n}$ are $(1, n)-$ quasihomogeneous polynomials of weighted degrees $i+1$ and $i+n$, respectively.

\section{Some CANONicAl FORMS FOR THE MONODROMIC NILPOTENT SINGUlARity}

In this section we will present some well known canonical forms for the monodromic nilpotent singularity. Also we will prove some properties that we will need later.

2.1. The Andreev canonical form. We consider system (1) and we assume that the origin is a nilpotent monodromic singular point with Andreev number $n$. Then, using the function $F$ of Theorem 1 we can define the analytic change of variables $(x, y) \mapsto(x, y-F(x))$. It is easy to see that this change transforms system (1) into

$$
\dot{x}=y+y \hat{P}(x, y), \quad \dot{y}=f(x)+y \varphi(x)+y^{2} \hat{Q}(x, y),
$$

where $\hat{P}(0,0)=0$ and the functions $f$ and $\varphi$ that appear here are exactly those as for (1). Finally, will be useful in future manipulations to make the rescaling of the coordinates given by $(x, y) \mapsto(\xi x,-\xi y)$ with $\xi=(-1 / a)^{1 /(2-2 n)}$ introducing a minus sign in the first equation of the differential system. The outcome is bringing system (1) into

$$
\dot{x}=y(-1+\tilde{P}(x, y)), \quad \dot{y}=\hat{f}(x)+y \hat{\varphi}(x)+y^{2} \tilde{Q}(x, y),
$$


where $P(0,0)=0, \hat{f}(x)=x^{2 n-1}+\cdots$ with $n \geq 2$ and either $\hat{\varphi}(x) \equiv 0$ or $\hat{\varphi}(x)=$ $b x^{\hat{\beta}}+\cdots$ with $\hat{\beta} \geq n-1$. Of course when $\hat{\beta}=n-1$ we also have that $b^{2}-4 n<0$. Hence the functions $\hat{f}$ and $\hat{\varphi}$ in (2) play for (2) the same roles as the functions $f$ and $\varphi$ described in Theorem 1. Although these couple of functions are different they have the same order at the origin, which means that the characteristic numbers $\hat{\beta}$ and $n$ in (1) are the same than in (2).

Remark 4. Clearly systems (1) and (2) are analytically conjugate and, as one can check from the specific conjugation, the Andreev number $n$ and the characteristic number $\hat{\beta}$ of (1) and (2) is unchanged. But it is easy to see that when (1) is analytically conjugate or analytically orbitally conjugate to another differential system, in general, $\hat{\beta}$ changes although the monodromic relation $\hat{\beta} \geq n-1$ must be invariant.

We will see that the Andreev number $n$ is, in fact, an invariant under analytic orbital conjugation.

Proposition 5. The Andreev number $n$ associated to an analytic (formal) monodromic nilpotent singularity is invariant under analytic (formal) orbital conjugation.

Proof. Let $\mathcal{X}=(y+P(x, y)) \partial_{x}+Q(x, y) \partial_{y}$ be the associated vector field to system (1) having a monodromic nilpotent singularity at the origin with Andreev number $n$. We know that there is a concrete analytic diffeomorphism $\Phi$ (the composition of $(x, y) \mapsto(x, y-F(x))$ with the scaling $(x, y) \mapsto(\xi x,-\xi y))$ such that the push forward $\Phi_{*} \mathcal{X}$ is just the vector field associated to (2). We write for simplicity $\Phi_{*}(\mathcal{X})=\mathcal{Y}=y \hat{P}(x, y) \partial_{x}+(f(x)+y \hat{Q}(x, y)) \partial_{y}$ with $f(x)$ having order $2 n-1$ at the origin.

Let $\mathcal{Z}$ be any vector field which is analytically orbitally conjugated to $\mathcal{X}$. In particular it is also analytically orbitally conjugated to $\mathcal{Y}$, that is, there is analytic diffeomorphism $\Psi$ in a neighborhood $U \subset \mathbb{R}^{2}$ of the origin of and an analytic function $\lambda: U \rightarrow \mathbb{R}$ with $\lambda(0,0) \neq 0$ such that $\mathcal{Z}=\lambda \Psi_{*}(\mathcal{Y})$. Now the structure of $\mathcal{Y}$ implies that $\mathcal{Z}=(y+\cdots) \partial_{x}+\left(f^{*}(x)+y Q^{*}(x, y)\right) \partial_{y}$ where the order of $f^{*}(x)$ at the origin is $2 n-1$. Since the monodromy of the origin is preserved under analytic orbital conjugation we conclude that the Andreev number of the origin for the vector field $\mathcal{Z}$ is $n$.

2.2. The Liénard canonical form. We recall here that Takens proved in [22] that a system with nilpotent linear part (be monodromic or not) can be formally transformed into a Liénard system $\dot{x}=-y, \dot{y}=a(x)+y \tilde{b}(x)$ with $a, \tilde{b} \in \mathbb{R}[[x]]$ formal power series such that $a(x)=a_{s} x^{s}(1+\mathcal{O}(x))$ with $s \geq 2$ and $\tilde{b}(0)=0$. Later, Strózyna and Zoladek show in [21] that indeed the former change can be choosen convergent. Furthermore, in the monodromic case, $s=2 n-1$ with $n \geq 2$ and after the change $x \mapsto u$ with $u(x)=\left(2 n \int_{0}^{x} a(z) d z\right)^{1 /(2 n)}=x\left(a_{2 n-1}+\mathcal{O}(x)\right)^{1 /(2 n)}$ and the time rescaling $t \mapsto \tau$ with $d t / d \tau=u^{2 n-1} / a(x)=a_{2 n-1}^{-1 /(2 n)}+\mathcal{O}(x)$ the above Liénard system is written as the analytic system

$$
\dot{x}=-y, \dot{y}=x^{2 n-1}+y b(x),
$$

where $b(x)=\sum_{j \geq \beta} b_{j} x^{j}$ and $b_{\beta} \neq 0$. Clearly, momodromy is preserved under orbital analytic conjugation, hence (3) satisfies one of the following conditions: (i) $\beta>n-1$; (ii) $\beta=n-1$ and $b_{\beta}^{2}-4 n<0$; (iii) $b(x) \equiv 0$. 
From (3) it is not difficult to characterize the center case. The origin of (3) is a center if and only if $b(x)$ is an odd function, see [5,20,23]. Also, as Strózyna and Żołądek prove in [21], the monodromic system (1) has a local analytic first integral if and only if $b(x) \equiv 0$.

2.3. Generalized polar coordinates. We recall that the generalized trigonometric functions used by Lyapunov [18] are the unique solution $x(\theta)=\operatorname{Cs} \theta$ and $y(\theta)=\operatorname{Sn} \theta$ of the Cauchy problem $\frac{d x}{d \theta}=-y, \frac{d y}{d \theta}=x^{2 n-1}$ with initial condition $(x(0), y(0))=(1,0)$. Now we introduce the the generalized polar blow-up $(x, y) \mapsto(r, \theta)$ defined by

$$
x=r \operatorname{Cs} \theta, \quad y=r^{n} \operatorname{Sn} \theta
$$

that embeds a neighborhood of the origin into a cylinder $C=\left\{(r, \theta) \in \mathbb{R} \times \mathcal{S}^{1}\right\}$ with $|r|$ sufficiently small. Performing the change (4), system (2) with Andreev number $n$ is transformed into an ordinary differential equation over the cylinder $C$ of the form:

$$
\frac{d r}{d \theta}=\mathcal{F}(r, \theta),
$$

where $\mathcal{F}(r, \theta)$ is an analytic function on $C$ and $\mathcal{F}(0, \theta) \equiv 0$ for all $\theta \in \mathcal{S}^{1}$, that is, the circle $\{r=0\}$ becomes a particular periodic solution of (5). We will take $\mathcal{S}^{1}=\mathbb{R} / T \mathbb{Z}$ where $T$ is the minimal period of both $\operatorname{Cs} \theta$ and $\operatorname{Sn} \theta$. More precisely in [18] it is proved that $T=2 \sqrt{\frac{\pi}{n}} \frac{\Gamma\left(\frac{1}{2 n}\right)}{\Gamma\left(\frac{n+1}{2 n}\right)}$, where $\Gamma(\cdot)$ denotes the Euler Gamma function. With more details (see for example $[2,9]$ ) we have

$$
\dot{r}=\mathcal{R}(r, \theta)=\tilde{p}(\theta) r^{n+1}+O\left(r^{n+2}\right), \quad \dot{\theta}=\Theta(r, \theta)=r^{n-1}+O\left(r^{n}\right),
$$

when $\hat{\beta} \geq n$ or $\varphi(x) \equiv 0$, and

$$
\begin{aligned}
& \dot{r}=\mathcal{R}(r, \theta)=\mathrm{Cs}^{n-1} \theta \operatorname{Sn}^{2} \theta r^{n}+O\left(r^{n+1}\right), \\
& \dot{\theta}=\Theta(r, \theta)=\left(1+b \operatorname{Cs}^{n} \theta \operatorname{Sn} \theta\right) r^{n-1}+O\left(r^{n}\right),
\end{aligned}
$$

when $\hat{\beta}=n-1$, in which case one can check that $1+b \operatorname{Cs}^{n} \theta \operatorname{Sn} \theta>0$ for any $\theta \in[0, T)$ due to Andreev's monodromic condition $4 n-b^{2}>0$. In summary (7) writes as

$$
\frac{d r}{d \theta}=\frac{\mathcal{R}(r, \theta)}{\Theta(r, \theta)}:=\mathcal{F}(r, \theta)= \begin{cases}\frac{O\left(r^{2}\right)}{1+O(r)} & \text { if } \hat{\beta} \geq n \text { or } \varphi(x) \equiv 0 \\ \frac{O(r)}{1+b \operatorname{Cs}^{n} \theta \operatorname{Sn} \theta+O(r)} & \text { if } \quad \hat{\beta}=n-1 .\end{cases}
$$

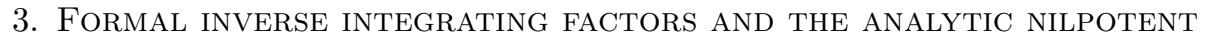
CENTER PROBLEM

3.1. Initial considerations. We recall that $V: U \subset \mathbb{R}^{2} \rightarrow \mathbb{R}$ is said to be an inverse integrating factor of system (1) in a neighborhood $U$ of the origin if it is of class $C^{1}(U)$, it is not locally null and it satisfies the linear partial differential equation

$$
\mathcal{X}(V)=V \operatorname{div} \mathcal{X}
$$

where $\mathcal{X}=(y+P(x, y)) \partial_{x}+Q(x, y) \partial_{y}$ is the associated vector field to system (1) and $\operatorname{div} \mathcal{X}$ its divergence. We refer to the survey paper [10] for a detailed explanation 
of several properties on inverse integrating factors. In particular it will be useful for us how inverse integrating factors change under orbital conjugation of vector fields.

Proposition 6. Let $\Phi$ be a diffeomorphism in $U$ with non-vanishing jacobian determinant $\operatorname{det}(D \Phi)$ on $U$ and let $\xi: U \rightarrow \mathbb{R}$ be such that $\xi \in C^{1}(U)$ and $\xi(0,0) \neq 0$. If $V$ is an inverse integrating factor of the vector field $\mathcal{X}$ in $U$ then $\xi(V \circ \Phi) / \operatorname{det}(D \Phi)$ is an inverse integrating factor of the orbitally conjugate vector field $\xi \Phi_{*}(\mathcal{X})$.

When there is a formal series $V \in \mathbb{R}[x, y]$ with $V \not \equiv 0$ that formally satisfies (8) we say that $V$ is a formal inverse integrating factor of $\mathcal{X}$. At this formal level, Proposition 6 also works if we replace $\Phi$ and $\xi$ by formal series. Therefore, the next proposition is a straightforward consequence.

Proposition 7. Let $\mathcal{X}$ and $\hat{\mathcal{X}}$ be two formally orbitally conjugate vector fields. Let $V(x, y)$ be a formal inverse integrating factor of $\mathcal{X}$ having the expansion $V(x, y)=$ $V_{s}+\sum_{j \geq s+1} V_{j}(x, y)$ where the $V_{j}$ are $(1, n)$-quasihomogeneous polynomials of weighted degree $j$ and $V_{s} \not \equiv 0$. Then there is a formal inverse integrating factor $\hat{V}$ of $\hat{\mathcal{X}}$ of the form $\hat{V}(x, y)=V_{s}+\sum_{j \geq s+1} \hat{V}_{j}(x, y)$ for some $(1, n)$-quasihomogeneous polynomials $\hat{V}_{j}$ of weighted degree $j$.

Clearly the existence of a formal inverse integrating factor $V(x, y)$ such that $V(0,0) \neq 0$ implies that one can construct an associated formal first integral of (1). Hence from the results in [19] the existence of a formal first integral implies the existence of a local analytic first integral (around any isolated singularity at the origin of any analytic planar vector field). Therefore we conclude that, if the origin is monodromic, it turns out to be a center.

On the contrary, there are analytic monodromic nilpotent singularities whose vector field has a formal inverse integrating factor $V$ but the origin is not a center. Clearly in such a case $V$ vanishes at the singularity. If $V$ is an inverse integrating factor of (1) in $U$ then the rescaled vector field $\mathcal{X} / V$ is Hamiltonian on $U \backslash V^{-1}(0)$ where $U \subset \mathbb{R}^{2}$ is a simply connected domain. Hence, denoting $H$ the Hamiltonian function,

$$
V(x, y)=\frac{y+P(x, y)}{\frac{\partial H}{\partial y}(x, y)}=-\frac{Q(x, y)}{\frac{\partial H}{\partial x}(x, y)} .
$$

From these expressions it is obvious that if (1) has an analytic first integral $H$ then it also has a meromorphic (quotient of analytic functions) inverse integrating factor. In fact, in the monodromic case we have more information as the next result shows (which is implicit in the work [1]).

Proposition 8. If there is a formal first integral of (1) with a monodromic point at the origin then (1) also has an analytic inverse integrating factor.

Proof. We first recall that Mattei and Moussu [19] proved that the existence of a formal first integral implies the existence of a local analytic first integral around any isolated singularity of any analytic planar vector field. Second, from a result in [21], we also known that a nilpotent center is locally analytically integrable if and only if it is analytically orbitally equivalent to the Hamiltonian system (3) with $b(x) \equiv 0$.

With these ingredients and recalling that $(3)$ with $b(x) \equiv 0$ has a polynomial inverse integrating factor, the proof is trivial. 
Remark 9. A source of formal planar vector fields with formal inverse integrating factor founded in [1] is the following. Let $H(x, y)$ and $\lambda(x, y)$ be $(1, n)$ quasihomogeneous polynomials of weighted degrees $p$ and $q$, respectively, and $g \in$ $\mathbb{R}[[z]]$ a formal series. Define the hamiltonian vector field $\mathcal{X}_{H}=-H_{y} \partial_{x}+H_{y} \partial_{y}$ and the Euler vector field $\mathcal{X}_{E}=x \partial_{x}+n y \partial_{y}$. Then $\mathcal{X}=\mathcal{X}_{H}+\lambda g(H) \mathcal{X}_{E}$ possesses the inverse integrating factor $V=H^{k} g(H)$ provided that $1+n-k p+q=0$.

Also in [1] it is proved that the vector field $\mathcal{X}$ associated with system (1) with $\hat{\beta} \geq n$ has a formal inverse integrating factor $V$ if and only if it is formally orbitally equivalent to

$$
\mathcal{X}_{H}+x^{n-1} f(H) \mathcal{X}_{E}
$$

with $H(x, y)=x^{2 n}+n y^{2}$ and $f(H)=\sum_{i \geq \ell} a_{i} H^{i}$ a certain formal series with $a_{\ell} \neq 0$. Clearly (1) is formally integrable if and only if $f \equiv 0$. Also, in case that $f \not \equiv 0$, one has $V=H^{\ell+1} g(H)$ with $g(H)=f(H) /\left(a_{\ell} H^{\ell}\right)=1+\cdots$.

3.2. Inverse integrating factors on the cylinder and the center problem. An inverse integrating factor of (5) is a function $\mathcal{V}: C \rightarrow \mathbb{R}$ of class $C^{1}(C)$, which is non-locally null and satisfies the partial differential equation:

$$
\frac{\partial \mathcal{V}(r, \theta)}{\partial \theta}+\frac{\partial \mathcal{V}(r, \theta)}{\partial r} \mathcal{F}(r, \theta)=\frac{\partial \mathcal{F}(r, \theta)}{\partial r} \mathcal{V}(r, \theta)
$$

Therefore, in particular, $\mathcal{V}(r, \theta)$ is a $T$-periodic function in the variable $\theta$.

If $V(x, y)$ is an inverse integrating factor of system (2) defined in a neighborhood of the nilpotent monodromic singularity at the origin with Andreev number $n$ then, using Proposition 6 and recalling that the jacobian determinant of the map (4) is $r^{n}$, it is evident that an inverse integrating factor $\mathcal{V}(r, \theta)$ of the corresponding equation (5) is

$$
\mathcal{V}(r, \theta)=\frac{V\left(r \operatorname{Cs} \theta, r^{n} \operatorname{Sn} \theta\right)}{r^{n} \Theta(r, \theta)} .
$$

Statement (i) of the following theorem is proved in [9] while the rest of the statements are some of the results of [11] with emphasis in the nilpotent case.

Theorem 10. Consider system (2) where the origin is a nilpotent monodromic singularity. Let $\mathcal{V}(r, \theta)$ be an inverse integrating factor of the corresponding equation (5) which has a Laurent expansion in a neighborhood of $r=0$ of the form

$$
\mathcal{V}(r, \theta)=\sum_{i \geq m} v_{i}(\theta) r^{i}
$$

with $v_{m}(\theta) \not \equiv 0$ and $m \in \mathbb{Z}$. We consider the function

$$
G(r)=\int_{0}^{T} \frac{\mathcal{F}(r, \theta)}{\mathcal{V}(r, \theta)} d \theta .
$$

Then the following holds:

(i) If $m \leq 0$ or $m+n$ is odd then the origin of system (2) is a center.

(ii) If the origin of system (2) is a center, then the function $G(r)$ is identically zero for all $r>0$ sufficiently small.

(iii) If $G(0)=0$ then the origin of system (2) is a center.

When an inverse integrating factor $\mathcal{V}(r, \theta)$ of (5) has an expansion (12) with $m \geq 1$, the integer $m$ is called the vanishing multiplicity of $\mathcal{V}$ at $r=0$. 
3.3. The Poincaré map. Let $\Psi\left(\theta ; r_{0}\right)$ be the solution of $(5)$ with initial condition $\Psi\left(0 ; r_{0}\right)=r_{0}$. Then we can define the Poincaré map $\Pi: \Sigma \subseteq \mathbb{R} \rightarrow \mathbb{R}$ as $\Pi\left(r_{0}\right)=$ $\Psi\left(T ; r_{0}\right)$. Thus $\Pi$ is an analytic diffeomorphism tangent to the identity and defined in a neighborhood $\Sigma$ of $r_{0}=0$ whose fixed points correspond to periodic orbits of (5). Clearly the origin of (1) is a center if and only if the Poincaré map is just the identity, that is, $\Pi\left(r_{0}\right)=r_{0}$. In [8] it is proved the following fundamental result

$$
\mathcal{V}\left(\Pi\left(r_{0}\right), 0\right)=\mathcal{V}\left(r_{0}, 0\right) \Pi^{\prime}\left(r_{0}\right)
$$

An interesting question is to know under what conditions over $\mathcal{V}$ the fundamental functional equation (14) only have the trivial solution $\Pi\left(r_{0}\right)=r_{0}$. We emphasize that from [8] (see also [9]) we known that a nontrivial solution $\Pi\left(r_{0}\right)=r_{0}+O\left(r_{0}^{2}\right)$ must be of the form

$$
\Pi\left(r_{0}\right)=r_{0}+c_{m} r_{0}^{m}+O\left(r_{0}^{m+1}\right)
$$

with $c_{m} \neq 0$ and $m$ is just the vanishing multiplicity of $\mathcal{V}$ at $r=0$.

\section{Main Results}

We started showing that the simplest formal inverse integrating factors of the simplest Liénard canonical form (3), namely the polynomial ones, cannot coexist except in just a trivial case.

Proposition 11. The only possible polynomial inverse integrating factor $V$ of a polynomial Liénard canonical form $(3)$ is $V(x, y)=y-c$ with $c \neq 0$ a constant, in which case $b(x)=x^{2 n-1} / c$.

Proof. Clearly the zero-set $V^{-1}(0) \subset \mathbb{R}^{2}$ is an invariant algebraic curve for the flow of (3). Then we can use the classification given in Theorem 2 of [24] of all the possible forms that an invariant algebraic curve can have in arbitrary polynomial Liénard differential systems (not necessarily of the form (3)). The three possibilities (rational, hyperelliptic or $d$-fold with $d \geq 3$ algebraic curves) are listed below.

(i) Following [24], the $d$-fold case only is possible if $\operatorname{deg}(b)=n-2$ which is in contradiction with our monodromic constraint $\operatorname{deg}(b) \geq \beta \geq n-1$.

(ii) Assume that $V(x, y)=(y+P(x))^{2}-Q(x)$, that is, $V^{-1}(0)$ is a hyperelliptic curve. In [24] it is proved that this can happen only if $\operatorname{deg}(b)<2 n-1$. Imposing the proposed expression of $V$ to be a solution of (8) and equation the coefficients of the three different powers of $y$ yields $P^{\prime}(x)=-b(x) / 2, Q^{\prime}(x)=-2 x^{2 n-1}-b(x) P(x)$, and the constraint $C(x):=b(x)\left(Q(x)-P^{2}(x)\right)-2 x^{2 n-1} P(x) \equiv 0$. Taking into account the orders $P(x)=O\left(x^{\beta+1}\right)$ and $Q(x)=O\left(x^{2 n-1}\right)$, it is straightforward to see after the analysis of the minimum degree in $C(x)$ that it must be $\beta+2 n-1=$ $3 \beta+2$, hence we arrive to the contradiction $\beta=n-3 / 2$.

(iii) Suppose that $V^{-1}(0)$ is a rational curve, that is, $V(x, y)=y-P(x)$. Imposing this $V$ satisfies the partial differential equation (8) gives $P^{\prime}(x) \equiv 0$ and $b(x)=x^{2 n-1} / P(x)$ finishing the proof.

The next theorem is one of the main results of this work. The first part was already proved in [1] via quasihomogeneous normal form theory in the special case $\hat{\beta} \geq n$. We will prove it using a different approach and for all the possible values of $\hat{\beta}$, hence also including the case $\hat{\beta}=n-1$. The second part characterizes the nilpotent centers with Andreev number $n=2$ via the existence of a formal inverse 
integrating factor. This result can be stated joining three works [15], [16] and [17] and we will prove it in a simpler and unified form.

Theorem 12. Consider system (1) where the Andreev number $n$ associated to the monodromic nilpotent singularity at the origin is even. If (1) possesses a formal inverse integrating factor then the origin is a center. Moreover if $n=2$ then the converse is also true.

Proof. The first part is a direct consequence of a result of [9]. In [9] it is proved that a necessary condition for having an analytic (with trivial extension to the formal setting) inverse integrating factor $V(x, y)$ defined in a neighborhood of a focus at the origin of the analytic system (1) is that its Andreev number $n$ must be odd. Therefore, if $n$ is even and there is such a formal $V(x, y)$ then the origin is a center. This proves the first part of the theorem.

Now we shall prove the second part. First, recall that system (1) with a nilpotent analytic monodromic singularity at the origin is analytically orbitally equivalent to the Liénard vector field (3). From now we assume that $n$ is even and the origin of (1) is a center. Recall that, from Proposition 5, $n$ remains unchanged under analytic orbital conjugation.

We thus have $b(x)=x B\left(x^{2}\right)$ with $B(u)$ an analytic function at $u=0$. In particular we see that $\beta$ is odd. Let $\bar{\beta}$ be the order at the origin of $B$, thus $B(u)=\sum_{j \geq \bar{\beta}} B_{j} u^{j}$ with $B_{\bar{\beta}}=b_{\beta} \neq 0$ and $\beta=2 \bar{\beta}+1$. Since $\beta \geq n-1$, we have $\bar{\beta} \geq(n-2) / 2 \geq 0$. In this case the polynomial mapping $(x, y) \mapsto(u, y)=\Psi(x, y)=$ $\left(x^{2}, y\right)$ yields, after rescaling the vector field by $1 / x$, a new Liénard system

$$
\dot{u}=-2 y, \quad \dot{y}=u^{n-1}+y B(u) .
$$

Let us first consider the simplest case $n=2$ and $\beta=n-1$. Then $B(0)=B_{0}=$ $b_{\beta} \neq 0$ yielding that the singularity at the origin of (16) is a strong focus since the associated eigenvalues are $\frac{1}{2}\left(b_{\beta} \pm \sqrt{b_{\beta}^{2}-8}\right)$ with $b_{\beta} \neq 0$ and $b_{\beta}^{2}-8<0$ from the monodromic conditions. Then there is an analytic inverse integrating factor $\hat{V}(u, y)$ near the origin (recall that a strong focus linearizes due to the nature of its spectrum).

If $n=2$ but $\beta \geq n$ then the origin of (16) has complex eigenvalues $\pm i \sqrt{2}$. Hence, the origin of (16) becomes a (nondegenerate) center or a weak focus depending on the nonlinearities $B(u)$. In the center case there is an analytic inverse integrating factor $\hat{V}(u, y)$ near the origin (Reeb's criterium). In the focus case there is a smooth (and non-flat at the origin, hence formal) inverse integrating factor $\hat{V}(u, y)$ as it is proved in [6].

In short, when $n=2$ and the origin is a center of (3) we have proved that always (16) has a formal inverse integrating factor $\hat{V}(u, y)$. The proof finishes by noticing that, in this situation, $\hat{V}\left(x^{2}, y\right)$ is a formal inverse integrating factor of $(3)$. This follows using how inverse integrating factors change under maps and time-rescalings (recall that $\operatorname{det}(D \Phi)=2 x$ ) as it is explained in Proposition 6. Also notice that (3) is time-reversible with respect to the involution $(x, y, t) \mapsto(-x, y,-t)$ and this forces that any formal inverse integrating factor of it must be an even function of the variable $x$. 
We end the argument just emphasizing that the existence of $\hat{V}\left(x^{2}, y\right)$ turns out to the existence of a formal inverse integrating factor of (1) due to the analytical orbital conjugation between (16) and (1).

The next proposition generalizes the second part of Theorem 12, which is included when $n=2$.

Proposition 13. Consider system (1) with a center at the origin and Andreev number $n=2^{k}$ for some positive $k \in \mathbb{N}$. Assume its associated Liénard system (3) has $b(x)=x^{n-1} B_{k}\left(x^{n}\right)$ for some function $B_{k}$ analytic at 0 . Then (1) possesses a formal inverse integrating factor.

Proof. We will follow the lines of the proof of the second part of Theorem 12. We want to explore under which conditions we can repeat several times (actually $k$ times) the fold $x \mapsto x^{2}$ and and the time reescaling by $1 / x$ to system (3) ending the process with an analytic system having a nilpotent monodromic singularity at the origin with Andreev number 2. We will see that these conditions are just the restriction on $n$ and $b(x)$ stated in hypothesis of the proposition.

Let $n=2^{k}$ for certain $k \in \mathbb{N}$. If $k=1$ then we have finished because the proof is just the second part of Theorem 12. If $k \geq 2$ then the origin of (16) becomes a nilpotent singularity which is monodromic only when $n$ is even (this is our situation), in which case has Andreev number $N=n / 2=2^{k-1}$. More specifically (16) is written as

$$
\dot{u}=-2 y, \quad \dot{y}=u^{2 N-1}+y B(u)
$$

with $B(u)$ having order $\bar{\beta} \geq N-1$ at the origin. The origin is again a center (because $B(u)$ is odd due to the assumptions on $b(x)$ ) and then we can repeat $k-1$ times the arguments $\left(u \mapsto v=u^{2}\right.$ and a time reescaling by $\left.1 / u\right)$ used in the second part of the proof of Theorem 12 until we reach 2 as Andreev number. In this procedure we get always a center at the origin along a cascade of even Andreev numbers. Then we can also conclude that (1) possesses a formal inverse integrating factor from Theorem 12 with $n=2$.

Remark 14. In the case $n$ odd, $\hat{\beta} \geq n$, and assuming the existence of a formal inverse integrating factor, the work [1] proves that the origin of (1) is a center if and only if it is formally integrable. The proof goes as follows. Taking generalized polar coordinates (4) the formal canonical form (9) is transformed into an equation (5) of the form

$$
\frac{d r}{d \theta}=\mathcal{F}(r, \theta)=-r \mathrm{Cs}^{n-1} \theta f\left(r^{2 n}\right)
$$

with $f \not \equiv 0$ if (1) is not formally integrable. Separating variables we get

$$
\int_{r_{0}}^{\Pi\left(r_{0}\right)} \frac{d r}{r f\left(r^{2 n}\right)}=-\int_{0}^{T} \operatorname{Cs}^{n-1} \theta d \theta,
$$

from where we see that the center case $\Pi\left(r_{0}\right)=0$ cannot be achieved when $n$ is odd.

Notice that this argument also proves the first part of Theorem 12 in the particular case $\hat{\beta} \geq n$ because when $n$ is even then the integral of the righthand side of (17) becomes zero (see [18]). 
Now we characterize up to a multiplicative constant the leading term in the $(1, n)$-quasihomogeneous expansion of any formal inverse integrating factor $V$ of (1) in case of having associated a Liénard normal form (3) with $\beta=n-1$. This case is not included in the analysis performed in [1].

Proposition 15. Consider system (1) with a monodromic point at the origin with Andreev number $n$. When $\beta=n-1$ in the Liénard normal form (3), if there is a formal inverse integrating factor $V$ of (1) then it is unique up to a multiplicative constant, and moreover it has the form $V(x, y)=x^{2 n}+n y^{2}+b_{n-1} x^{n} y+$ $\sum_{j \geq 2 n+1} V_{j}(x, y)$ where the $V_{j}$ are $(1, n)$-quasihomogeneous polynomials of weighted degree $j$ and $b_{n-1} \in \mathbb{R} \backslash\{0\}$.

Proof. Recall that a monodromic system (1) is analytically orbitally equivalent to the Liénard vector field (3). Therefore, the formal inverse integrating factors of (1) and (3) are in one-to-one correspondence and, moreover, each pair has the same order at the origin. So, it is enough to performing the analysis of (3).

Let us write the analytic system (3) as

$$
\dot{x}=\sum_{i \geq n} p_{i}(x, y), \dot{y}=\sum_{i \geq 2 n-1} q_{i}(x, y) \text {, }
$$

where $p_{i}, q_{i}$ are $(1, n)$-quasihomogeneous polynomials of weighted degree $i$ and

$$
p_{n}(x, y)=-y, q_{2 n-1}(x, y)= \begin{cases}x^{2 n-1} & \text { if } \beta \geq n \text { or } \varphi(x) \equiv 0 \\ x^{2 n-1}+b_{n-1} y x^{n-1} & \text { if } \beta=n-1\end{cases}
$$

In other words, if we let $\mathcal{X}$ to denote the associated vector field to system (3), then $\mathcal{X}=\sum_{i \geq n-1} \mathcal{X}_{i}$ where $\mathcal{X}_{i}$ denotes a $(1, n)$-quasihomogeneous polynomial vector field of weighted degree $i$. Of course $\mathcal{X}_{n-1}=p_{n}(x, y) \partial_{x}+q_{2 n-1}(x, y) \partial_{y}$.

Let $V(x, y)$ be a formal inverse integrating factor of system (3). Hence $V$ has the expansion $V(x, y)=\sum_{i>s} V_{i}(x, y)$ where $V_{i}$ is a $(1, n)$-quasihomogeneous polynomial of weighted degree $i$. Imposing $\mathcal{X}(V)=V \operatorname{div} \mathcal{X}$ and taking its lower $(1, n)$ quasihomogeneous terms (which are just of weighted degree $n+s-1$ ) we get

$$
\mathcal{X}_{n-1} V_{s}=V_{s} \operatorname{div} \mathcal{X}_{n-1}
$$

This means that $V_{s}(x, y)$ is a polynomial inverse integrating factor of $\mathcal{X}_{n-1}$. We recall that $\mathcal{X}_{n-1}$ always possesses the polynomial inverse integrating factor $V_{2 n}^{*}(x, y)=$ $x q_{2 n-1}(x, y)-n y p_{n}(x, y)$ due to the quasi-homogeneity (use the generalized Euler theorem for quasihomogeneous functions $x \partial_{x} H_{s}+n y \partial_{y} H_{s}=s H_{s}$ ). Also it is well known that any other polynomial inverse integrating factor $V_{s}(x, y)$ of $\mathcal{X}_{n-1}$ is necessarily of the form $V_{s}(x, y)=V_{2 n}^{*}(x, y) H_{s-2 n}(x, y)$ where $H_{s-2 n}(x, y)$ is a a first integral of $\mathcal{X}_{n-1}$ which is either polynomial or rational with denominator $V_{2 n}^{*}(x, y)$. From here we easily deduce the following properties.

(i) Let $\beta=n-1$ (so that $b \neq 0$ ). If $n$ is even, then the origin is a nilpotent focus of $\mathcal{X}_{n-1}$. In consequence it has not rational first integral. This forces that $\mathcal{X}_{n-1}$ only has $V_{2 n}^{*}(x, y)=x^{2 n}+n y^{2}+b_{n-1} x^{n} y$ as linearly independent polynomial inverse integrating factor. On the contrary, when $n$ is odd the origin is a nilpotent center of $\mathcal{X}_{n-1}$. In order to know under what conditions $\mathcal{X}_{n-1}$ has a rational first integral we can use the results developed in [14]. 
The conclusion is that $\mathcal{X}_{n-1}$ has a rational first integral if and only if

$$
b_{n-1}^{2}-4 n \neq 0 \text { and } \frac{b_{n-1}}{\sqrt{b_{n-1}^{2}-4 n}} \in \mathbb{Q} \text {. }
$$

But the second condition cannot be possible since $b_{n-1}^{2}-4 n<0$ by monodromy. As a consequence we get again that $V_{2 n}^{*}(x, y)$ is the only linearly independent polynomial inverse integrating factor that possesses $\mathcal{X}_{n-1}$.

(ii) If $\beta \geq n$ then $\mathcal{X}_{n-1}$ is Hamiltonian (with first integral $H_{2 n}^{*}(x, y)=x^{2 n}+$ $\left.n y^{2}\right)$ and has a center at the origin. This implies that any linearly independent polynomial inverse integrating factor of $\mathcal{X}_{n-1}$ has the form $V_{2 k n}(x, y)=$ $\left(x^{2 n}+n y^{2}\right)^{k}$ with $k \in \mathbb{N} \cup\{0\}$.

In summary, (i) and (ii) implies that when $\beta=n-1$ in (3), any formal inverse integrating factor $V$ of (3), and consequently of (1), has the form $V(x, y)=$ $x^{2 n}+n y^{2}+b_{n-1} x^{n} y+\sum_{j \geq 2 n+1} V_{j}(x, y)$ where the $V_{j}$ are $(1, n)$-quasihomogeneous polynomials of weighted degree $j$. Moreover, it is unique up to multiplicative constants. The proof finishes after applying Proposition 7.

The next result is a byproduct of the proof of Proposition 15 combined with Proposition 6 and Proposition 7.

Corollary 16. Let $V(x, y)$ be a formal inverse integrating factor of (1) with a monodromic point at the origin having Andreev number $n$. Then there is an integer $k \geq 0$ such that, up to a multiplicative constant,

$$
V(x, y)=\left(x^{2 n}+n y^{2}+b_{n-1} x^{n} y\right)^{k}+\sum_{j \geq 2 k n+1} V_{j}(x, y)
$$

where the $V_{j}$ are $(1, n)$-quasihomogeneous polynomials of weighted degree $j$. Moreover the following holds:

(i) If $k=0$ or $k \geq 2$ then $b_{n-1}=0$.

(ii) If $b_{n-1} \neq 0$ then $k=1$ and $V$ is unique up to multiplicative constants.

Now it is straightforward to compute the set of possible values of the associated vanishing multiplicity $m$ of any formal $V$ of (1). As we see below, the minimum is reached at $m=1$

Corollary 17. If (1) has a formal inverse integrating factor $V(x, y)$, then the corresponding inverse integrating factor $\mathcal{V}(r, \theta)$ of (5) given by (11) has either leading exponent $m=1-2 n<0$ or vanishing multiplicity $m$ at $r=0$ given by $m=2(k-1) n+1$ for some integer $k \geq 1$.

Proof. From Corollary 16 we known that $V(x, y)=\sum_{j \geq 2 k n} V_{j}(x, y)$ where the $V_{j}$ are $(1, n)$-quasihomogeneous polynomials of weighted degree $j$ and $k \geq 0$ is an integer. Clearly, since $V_{2 k n} \not \equiv 0$ and the order at $r=0$ of $\Theta(r, \theta)$ is just $n-1$ we see that the corresponding $\mathcal{V}(r, \theta)$ given in (11) has an expansion (12) with $m=2(k-1) n+1$.

The next proposition characterizes the leading term in the $(1, n)$-quasihomogeneous expansion of any formal inverse integrating factor $V$ of (1) when its associated a Liénard normal form (3) has $\beta \geq n$ for certain values of $\beta$. 
Proposition 18. Let us consider system (1) having a monodromic point at the origin with Andreev number $n$ and its associated Liénard normal form (3) with $\beta \geq n$. Assume that (1) has a formal inverse integrating factor $V(x, y)$. Then either $V(0,0) \neq 0$ or, up to a multiplicative constant, $V(x, y)=x^{2 n}+n y^{2}+$ $\sum_{j \geq 2 n+1} V_{j}(x, y)$ where the $V_{j}$ are $(1, n)$-quasihomogeneous polynomials of weighted degree $j$ when one of the following conditions holds:

(i) $n \leq \beta \leq 2 n-1$;

(ii) $\beta$ is even and $\beta \neq(2 k-1) n-1$ with $k \geq 2$ a positive integer.

Proof. We follow the notation of the proof of Proposition 15.

Assume $\beta \geq n$. Then $\mathcal{X}_{n-1}=-y \partial_{x}+x^{2 n-1} \partial_{y}, \mathcal{X}_{j} \equiv 0$ for $j=n, \ldots, \beta-1$ and $\mathcal{X}_{\beta}=b_{\beta} y x^{\beta} \partial_{y}$ with $b_{\beta} \neq 0$. Recall that $\operatorname{div} \mathcal{X}=b(x)=b_{\beta} x^{\beta}+\cdots$. The equation $\mathcal{X} V=V \operatorname{div} \mathcal{X}$ is expanded into

$$
\left(\mathcal{X}_{n-1}+\mathcal{X}_{\beta}+\cdots\right)\left(V_{2 k n}^{*}+\sum_{i \geq 2 k n+1} V_{i}\right)=\left(V_{2 k n}^{*}+\sum_{i \geq 2 k n+1} V_{i}\right)\left(b_{\beta} x^{\beta}+\cdots\right)
$$

where from the proof of Proposition 15 we know that $V_{2 k n}^{*}(x, y)=\left(x^{2 n}+n y^{2}\right)^{k}$ for some $k \in \mathbb{N} \cup\{0\}$. Now we will explore the next $(1, n)$-quasihomogeneous terms. First we observe that $\mathcal{X}_{n-1}\left(V_{i}\right)=0$ for $2 k n+1 \leq i \leq r-1$ and

$$
\mathcal{X}_{n-1}\left(V_{r}\right)=F
$$

where $r=(2 k-1) n+\beta+1$, and

$$
F=b_{\beta} x^{\beta} V_{2 k n}^{*}-\mathcal{X}_{\beta}\left(V_{2 k n}^{*}\right)=b_{\beta} x^{\beta} V_{2(k-1) n}^{*}\left(V_{2 n}^{*}-2 n k y^{2}\right) .
$$

From this last equation and the Euler equation $\mathcal{X}_{E} V_{r}=r V_{r}$ with $\mathcal{X}_{E}=x \partial_{x}+n y \partial_{y}$ we can express

$$
\begin{aligned}
\frac{\partial V_{r}}{\partial x} & =\frac{r V_{r} x^{2 n-1}}{V_{2 n}^{*}}-b_{\beta} n V_{2(k-2) n}^{*} x^{\beta} y\left(V_{2 n}^{*}-2 n k y^{2}\right), \\
\frac{\partial V_{r}}{\partial y} & =\frac{r V_{r} y}{V_{2 n}^{*}}+b_{\beta} x^{\beta+1} V_{2(k-2) n}^{*}\left(V_{2 n}^{*}-2 n k y^{2}\right) .
\end{aligned}
$$

Therefore either $k \in\{0,1\}$ or $V_{2 n}^{*}$ divides $V_{r}$. We continue assuming the second option until we arrive to a contradiction when we take the hypothesis (i) or (ii) of the theorem.

So let $V_{r}=\left(V_{2 n}^{*}\right)^{\ell} W_{d}=V_{2 \ell n}^{*} W_{d}$ for some positive integer $\ell$ and $W_{d}$ is a $(1, n)$ quasihomogeneous polynomial of weighted degree $d=r-2 \ell n$ such that $V_{2 n}^{*}$ is not a factor of it. More precisely $W_{d}$ satisfies $V_{2 \ell n}^{*} \mathcal{X}_{n-1}\left(W_{d}\right)=F$ from where we see that $V_{2 \ell n}^{*}$ must divide $F$ and therefore $\ell \leq k-1$. Thus $\mathcal{X}_{n-1}\left(W_{d}\right)=G$ with $G=b_{\beta} x^{\beta} V_{2(k-1-\ell) n}^{*}\left(V_{2 n}^{*}-2 n k y^{2}\right)$. Repeating arguments with the former equation together with the Euler equation for $W_{d}$ yield

$$
\begin{aligned}
\frac{\partial W_{d}}{\partial x} & =-\frac{d W_{d} x^{2 n-1}}{V_{2 n}^{*}}-b_{\beta} n V_{2(k-2-\ell) n}^{*} x^{\beta} y\left(V_{2 n}^{*}-2 n k y^{2}\right), \\
\frac{\partial W_{d}}{\partial y} & =\frac{d W_{d} y}{V_{2 n}^{*}}+b_{\beta} x^{\beta+1} V_{2(k-2-\ell) n}^{*}\left(V_{2 n}^{*}-2 n k y^{2}\right) .
\end{aligned}
$$

Since $V_{2 n}^{*}$ does not divide $W_{d}$, it follows that $\ell=k-1$ which implies $d=\beta+n+1$. Now $W_{d}$ satisfies

$$
\mathcal{X}_{n-1}\left(W_{d}\right)=G, \quad \text { with } G=b_{\beta} x^{\beta}\left(x^{2 n}+n(1-2 k) y^{2}\right) .
$$


To prove statement (i) we observe that, from (21),

$$
\frac{\partial W_{d}}{\partial x}=x^{M} \Omega_{d-M-1}(x, y), \quad M=\min \{\beta, 2 n-1\}
$$

with $\Omega_{d-M-1}$ a $(1, n)$-quasihomogeneous polynomial of weighted degree $d-M-1$.

If $\beta \leq 2 n-1$ (the hypothesis of (i)) then $M=\beta$ and $d-M-1=n$. This means that $\Omega_{n}(x, y)=a_{0} x^{n}+a_{1} y$ with $a_{i} \in \mathbb{R}$ and

$$
W_{d}(x, y)=\int x^{\beta} \Omega_{n}(x, y) d x+f(y)
$$

where $f(y) \equiv 0$ if $2 n$ does not divide $d=\beta+n+1$ and $f(y)=a_{2} y^{d /(2 n)}$ otherwise. Inserting the obtained expression of $W_{d}$ into its defining equation (21) and next equating the coefficients of the monomials in both sides gives $a_{0}=a_{2}=0$, $a_{1}=b_{\beta}(\beta+1)$, and $\beta=n(2 k-1)-1$. This last expression of $\beta$ is incompatible with the assumed bound $n \leq \beta \leq 2 n-1$ for any $k \in \mathbb{N}$ and therefore we get a contradiction. This implies statement (i).

To prove statement (ii) we shall transform the partial differential equation (21) to generalized polar coordinates. First we have that $\mathcal{X}_{n-1}=r^{n-1} \partial_{\theta}$ and

$$
\hat{G}(r, \theta)=G\left(r \operatorname{Cs} \theta, r^{n} \operatorname{Sn} \theta\right)=b_{\beta} r^{\beta+2 n} \operatorname{Cs}^{\beta} \theta\left(1-2 k n \operatorname{Sn}^{2} \theta\right) .
$$

Therefore, the function $\hat{W}_{d}(r, \theta)=W_{d}\left(r \operatorname{Cs} \theta, r^{n} \operatorname{Sn} \theta\right)=r^{d} A_{d}(\theta)$, where $A_{d}$ is a $(1, n)$-quasihomogeneous trigonometric polynomial in $(\operatorname{Cs} \theta, \operatorname{Sn} \theta)$ of degree $d$, satisfies the equation

$$
\frac{\partial \hat{W}_{d}}{\partial \theta}=b_{\beta} r^{\beta+n+1} \mathrm{Cs}^{\beta} \theta\left(1-2 k n \operatorname{Sn}^{2} \theta\right) .
$$

Doing the quadrature yields

$$
\hat{W}_{d}(r, \theta)=b_{\beta} r^{\beta+n+1}\left[I_{1}(\theta)-2 k n I_{2}(\theta)\right]
$$

where

$$
I_{1}(\theta)=\int_{0}^{\theta} \mathrm{Cs}^{\beta}(s) d s, \quad I_{2}(\theta)=\int_{0}^{\theta} \mathrm{Cs}^{\beta}(s) \operatorname{Sn}^{2}(s) d s .
$$

Using the properties of the generalized trigonometric functions (explained in [18]) we get

$$
I_{2}(\theta)=\frac{1}{\beta+n+1}\left(I_{1}(\theta)-\operatorname{Sn} \theta \mathrm{Cs}^{\beta+1} \theta\right) .
$$

Therefore

$$
\begin{aligned}
A_{\beta+n+1}(\theta) & =b_{\beta}\left[I_{1}(\theta)-2 k n I_{2}(\theta)\right] \\
& =\frac{b_{\beta}}{\beta+n+1}\left[(\beta+(1-2 k) n+1) I_{1}(\theta)+2 k n \operatorname{Sn} \theta \mathrm{Cs}^{\beta+1} \theta\right],
\end{aligned}
$$

which implies that, either $\beta=(2 k-1) n-1$ with $k \geq 2$ or $I_{1}(\theta)$ is a $(1, n)$ quasihomogeneous trigonometric polynomial in $(\operatorname{Cs} \theta, \operatorname{Sn} \theta)$ of degree $\beta+n+1$. We will check that the second option is not possible when $\beta$ is even and this will prove statement (ii). 
Using again [18] yield

$$
\begin{aligned}
I_{1}(\theta)=\int_{0}^{\theta} \operatorname{Cs}^{\beta}(s) d s, & =\frac{n \operatorname{Sn} \theta \mathrm{Cs}^{\beta-2 n+1} \theta}{\beta-n+1}+\frac{\beta-2 n+1}{\beta-n+1} \int_{0}^{\theta} \operatorname{Cs}^{\beta-2 n}(s) d s, \\
\int_{0}^{\theta} \operatorname{Cs}^{2 n-1}(s) d s & =\operatorname{Sn} \theta .
\end{aligned}
$$

Thus we can get explicit expressions of $I_{1}(\theta)$ in terms of the functions $\xi_{r}(\theta)=$ $\int_{0}^{\theta} \operatorname{Cs}^{r}(s) d s$ with $1 \leq r \leq 2 n-2$. Actually, see [12], there exists a polynomial $E(x, y)$ and real constants $A$ and $B$ such that

$$
I_{1}(\theta)=\int_{0}^{\theta} \mathrm{Cs}^{\beta}(s) d s=E(\operatorname{Cs} \theta, \operatorname{Sn} \theta)+A \xi_{r}(\theta)+B \theta
$$

with $B \neq 0$ if and only if $\beta$ is even. Thus when $\beta$ is even $I_{1}(\theta)$ cannot be a $(1, n)$-quasihomogeneous trigonometric polynomial of the desired form.

A complement to statement (i) in Theorem 10 extending it to the case $m=1$ is presented in the following result. It essentially means that existence formal inverse integrating factor with the minimal associated vanishing multiplicity implies (generically) that (1) has a center.

Theorem 19. Consider system (1) with Andreev number $n$ associated to the monodromic nilpotent singularity at the origin and possessing $\hat{\beta} \geq n$. If there is a formal inverse integrating factor $V(x, y)=\sum_{j \geq 2 n} V_{j}(x, y)$ where the $V_{j}$ are $(1, n)$ quasihomogeneous polynomials of weighted degree $j$ and $V_{2 n} \not \equiv 0$ then the origin is a center. The converse is not true.

Proof. Clearly we can assume that (1) is written as (2) in the proof. If $V(x, y)$ is a formal inverse integrating factor of (2) with Andreev number $n$, then an inverse integrating factor $\mathcal{V}(r, \theta)$ of the corresponding equation (5) is given by (11) where $\Theta(r, \theta)$ is the function defined in (6) and has order exactly $n-1$ at $r=0$. Since $V\left(r \operatorname{Cs} \theta, r^{n} \operatorname{Sn} \theta\right)=\sum_{i \geq 2 n} \bar{v}_{i}(\theta) r^{i}$ with $\bar{v}_{i}(\theta) \not \equiv 0$ we obtain that $\mathcal{V}(r, \theta)$ has vanishing multiplicity $m=1$. Using now that the order of $\mathcal{F}(r, \theta)$ at $r=0$ is just 2 because $\hat{\beta} \geq n$ or $\varphi(x) \equiv 0$, we deduce that $\mathcal{F}(r, \theta) / \mathcal{V}(r, \theta)$ possesses order 1 at $r=0$ and therefore $G(0)=0$ where $G$ is defined by (13). Applying statement (iii) of Theorem 10 we conclude that the origin of (1) is a center.

The proof that the converse is false follows after the forthcoming counterexample (23) given in the section of examples.

Remark 20. In Theorem 19, the condition $\hat{\beta} \geq n$ is essential as the following example shows. Take the polynomial system (3) with $b(x)=b_{n-1} x^{n-1}$ so that $\hat{\beta}=\beta=n-1$. Hence this is a $(1, n)$-quasihomogeneous system of degree $n-1$ and, in consequence, it possesses the polynomial inverse integrating factor $V(x, y)=$ $V_{2 n}(x, y)=x^{2 n}+n y^{2}+b_{n-1} x^{n} y$. But clearly this system has a focus at the origin when $n$ is odd.

The next result is a corollary after combining Proposition 18 with Theorem 19 .

Corollary 21. Consider system (1) with a monodromic point at the origin with Andreev number $n, \hat{\beta} \geq n$, and its associated Liénard normal form (3) with $\beta \geq n$. If (1) has a formal inverse integrating factor and either 
(i) $n \leq \beta \leq 2 n-1$; or

(ii) $\beta$ is even and $\beta \neq(2 k-1) n-1$ with $k \geq 2$ a positive integer;

then (1) has a center at the origin.

Remark 22. In practice (for example when using Proposition 15, Proposition 18 or Corollary 21) if we only want to know which is the value of $\beta$ in the Liénard normal form (3) of (1), it is not necessary to get the complete normal form (3). It is sufficient if we can transform system $(1)$ into a $(1, n)$-quasihomogeneous orbital normal form up to order $n+r$ for a suitable $r \in \mathbb{N}$ such that $r \geq n-1$ of the form

$\dot{x}=-y+\mathcal{O}_{(1, n)}\left(|(x, y)|^{n+r}\right), \quad \dot{y}=x^{2 n-1}(1+\mathcal{O}(x))+y \bar{\varphi}(x)+\mathcal{O}_{(1, n)}\left(|(x, y)|^{n+r}\right)$,

with $\bar{\varphi}(x)=\bar{b}_{r} x^{r}+\mathcal{O}\left(x^{r+1}\right)$ and $\bar{b}_{r} \neq 0$. Here $\mathcal{O}_{(1, n)}\left(|(x, y)|^{k}\right)$ denotes a function whose $(1, n)$-quasihomogeneous expansion has leading term of weighted degree at least $k$. Hence we have proved that (3) has $\beta=r$.

\section{EXAMPLES}

5.1. Nilpotent center perturbed with a quasihomogeneous vector field. The following example was given in [9]. Consider $m, n \in \mathbb{N}$, positive integers with the relations $n \geq 2$ and $m \geq 1$. We associate to this pair the following system

$$
\dot{x}=y+x R(x, y), \quad \dot{y}=-x^{2 n-1}+n y R(x, y),
$$

where $R(x, y)$ is a $(1, n)$-quasihomogeneous polynomial of weighted degree $m+n-2$. The origin of system (22) is a nilpotent monodromic singularity with Andreev number $n$. Transforming it with generalized polar coordinates yields

$$
\dot{r}=r^{m+n-1} R(\operatorname{Cs} \theta, \operatorname{Sn} \theta), \quad \dot{\theta}=r^{n-1} .
$$

From here, separating variables, it is straightforward to see that (22) has a nilpotent center at the origin if and only if

$$
\int_{0}^{T} R(\operatorname{Cs} \theta, \operatorname{Sn} \theta) d \theta=0 .
$$

An inverse integrating factor is $\mathcal{V}(r, \theta)=r^{m}$ and easy computations (using the Euler theorem for quasihomogeneous functions) show that

$$
V(x, y)=\left(x^{2 n}+n y^{2}\right)^{\frac{m-1}{2 n}+1}
$$

is an inverse integrating factor of (22). Notice how the arbitrariness of $m$ and $n$ allows that $V(x, y)$ can be nonanalytic in a neighborhood of the origin. The analyticity of $V$ is achieved if, and only if, there exists an integer $k \geq 0$ such that $m=2 k n+1$, which is an odd integer.

Since $R(x, y)=r_{0} x^{m+n-2}+r_{1} x^{m-2} y+O\left(y^{2}\right)$ for some $r_{i} \in \mathbb{R}$, we can check by simple computations that $n$ is the Andreev number and either $\hat{\beta}=m+n-2$ if $r_{0} \neq 0$ or $\varphi(x) \equiv 0$ when $r_{0}=0$.

In summary, if $m+n$ is odd then the origin is a center. Also we see that there are both centers and foci coexisting with an analytic inverse integrating factor. In this last case we deduce that for having a center $n$ needs to be an even integer. 
5.2. A nilpotent center without formal inverse integrating factor. In [1] it is proved that system

$$
\dot{x}=y+x^{6}, \quad \dot{y}=-x^{7}+4 y x^{5},
$$

does not have a formal inverse integrating factor but it is a center since the origin is monodromic and the system is time-reversible (invariant with respect to the involution $(x, y, t) \mapsto(-x, y,-t))$.

We notice that at the origin this system possess the Andreev number $n=4$, $\hat{\beta}=5$ and moreover it is a particular case of system (22).

5.3. A nilpotent focus with polynomial inverse integrating factor. This example is extracted from [1]. The system $\dot{x}=y+x^{3} f(x, y), \dot{y}=-x^{5}+3 y x^{2} f(x, y)$ with $f(x, y)=x^{6}+3 y^{2}$ has $V(x, y)=f^{2}(x, y)$ as inverse integrating factor and the origin is a focus.

It is easy to check that the Andreev number is $n=3, \hat{\beta}=8$ and this system is a particular case of $(22)$.

5.4. Nilpotent center without formal first integral possessing formal inverse integrating factor. Consider the monodromic Liénard nilpotent canonical form (3) with the following conditions:

(i) $b(x) \not \equiv 0$ so that (3) does not have an analytic first integral;

(ii) $b(-x)=-b(x)$, hence (3) has a center at the origin;

(iii) Take the Andreev number $n=2$, which implies that (3) has a formal inverse integrating factor from (ii) and our Theorem 12.

\section{REFERENCES}

[1] A. Algaba, C. García and M. Reyes, Existence of an inverse integrating factor, center problem and integrability of a class of nilpotent systems, Chaos Solitons Fractals 45 (2012), 869-878.

[2] M.J. Álvarez and A. Gasull, Monodromy and stability for nilpotent critical points, Internat. J. Bifur. Chaos Appl. Sci. Engrg. 15 (2005), 1253-1265.

[3] M.J. Álvarez And A. Gasull, Generating limit cycles from a nilpotent critical point via normal forms, J. Math. Anal. Appl. 318 (2006), 271-287.

[4] A. ANDREEv, Investigation on the behaviour of the integral curves of a system of two differential equations in the neighborhood of a singular point, Translations Amer. Math. Soc. 8 (1958) 187-207.

[5] M. Berthier \& R. Moussu, Réversibilité et classification des centres nilpotents, Ann. Inst. Fourier (Grenoble) 44 (1994), 465-494.

[6] A. Enciso And D. Peralta-Salas, Existence and vanishing set of inverse integrating factors for analytic vector fields, Bull. London Math. Soc., 41 (2009), 1112-1124.

[7] I.A. García, H. Giacomini, J. Giné and J. Llibre, Analytic nilpotent centers as limits of nondegenerate centers revisited, to appear.

[8] I.A. García, H. Giacomini and M. Grau, The inverse integrating factor and the Poincaré map, Trans. Amer. Math. Soc., 362 (2010), 3591-3612.

[9] I.A. García, H. Giacomini and M. Grau, Generalized Hopf bifurcation for planar vector fields via the inverse integrating factor, J. Dyn. Differ. Equ. 23 (2011), 251-281.

[10] I.A. García and M. Grau, A survey on the inverse integrating factor, Qual. Theory Dyn. Syst., 9 (2010), 115-166.

[11] I.A. GARCía AND S. MAZA, A new approach to center conditions for simple analytic monodromic singularities, J. Differential Equations 248 (2010) 363-380.

[12] A. Gasull And J. Torregrosa, A new algorithm for the computation of the Lyapunov constants for some degenerated critical points, Nonlinear Anal. 47 (2001), 4479-4490. 
[13] H. Giacomini, J. Giné And J. Llibre, The problem of distinguishing between a center and a focus for nilpotent and degenerate analytic systems, J. Differential Equations 227 (2006) 406-426.

[14] J. Giné, M. Grau, And J. Llibre, Polynomial and rational first integrals for planar quasihomogeneous polynomial differential systems, Discrete Contin. Dyn. Syst. 33 (2013), 45314547.

[15] Y. LiU AND J. LI, New study on the center problem and bifurcations of limit cycles for the Lyapunov system (I), Internat. J. Bifur. Chaos 19 (2009), 3791-3801.

[16] Y. LiU AND J. LI, New study on the center problem and bifurcations of limit cycles for the Lyapunov system (II), Internat. J. Bifur. Chaos 19 (2009), 3087-3099.

[17] Y. LIU AND J. LI, On third-order nilpotent critical points: Integral factor method, Internat. J. Bifur. Chaos 21 (2011), 1293-1309.

[18] A. M. Lyapunov, Stability of motion. Mathematics in Science and Engineering, Vol 30. Academic Press, New York-London (1966).

[19] J.F. Mattei And R. Moussu, Holonomie et intégrales premières, Annales Scientifiques de l'École Normale Supérieure 13 (1980) 469-523.

[20] R. Moussu, Symétrie et forme normale des centres et foyers dégénérés, Ergodic Theory \& Dynam. Systems 2 (1982), 241-251.

[21] E. Strózyna \& H. ŻOŁA̧DEK, The analytic and formal normal form for the nilpotent singularity. J. Differential Equations 179 (2002), no. 2, 479-537.

[22] F. TAKens, Singularities of vector fields. Inst. Hautes tudes Sci. Publ. Math. 43 (1974), 47-100.

[23] M. A. TEIXEIRA \& J. YANG, The center-focus problem and reversibility, J. Differential Equations 174 (2001), 237-251.

[24] H. ŻoŁA̧DEK, Algebraic invariant curves for the Liénard equation, Trans. Amer. Math. Soc. 350 (1998), 1681-1701.

1 Departament de Matemàtica, Universitat de Lleida, Avda. Jaume II, 69, 25001 LLEIDA, SPAIN

E-mail address: garcia@matematica.udl.cat 